\title{
Les voix de Gobbo ou le nécessaire détour par l'absurde
}

\section{Claudine Defaye}

\section{(2) OpenEdition}

\section{Journals}

\section{Édition électronique}

URL : http://journals.openedition.org/shakespeare/363

DOI : 10.4000/shakespeare.363

ISSN : 2271-6424

\section{Éditeur}

Société Française Shakespeare

\section{Édition imprimée}

Date de publication : 1 novembre 1999

Pagination : 119-130

ISBN : 2-84269-331-0

\section{Référence électronique}

Claudine Defaye, «Les voix de Gobbo ou le nécessaire détour par l'absurde », Actes des congrès de la Société française Shakespeare [En ligne], 17 | 1999, mis en ligne le 01 novembre 2007, consulté le 22 avril 2019. URL : http://journals.openedition.org/shakespeare/363 ; DOI : 10.4000/shakespeare.363 


\section{S H A K E S P E A R E \\ \& $\quad$ L $A \quad$ V O I X}

Société Française Shakespeare

Actes du Congrès de 1999

米米

Textes réunis et présentés par

Patricia DORVAL

publiés sous la direction de

Jean-Marie MAGUIN 


\section{E S VOIX DE G O B B O O U LE NÉCESSAIRE DÉ T O UR PAR L'ABSURDE}

Le monologue de Lancelot Gobbo qui débute la cinquième scène du Marchand de Venise (II.2.1-30), complété par la tirade où il exprime son sentiment de triomphe (149-60) parce qu'il vient de prouver à ceux qui en auraient douté qu'il a bien une voix et un organe vocal fonctionnel «a tongue in [his] head» (150), peut paraître à première vue comme une sorte de détour verbal voire verbeux dont on pourrait peut-être faire l'économie ${ }^{1}$. Il est vrai que les deux mondes complémentaires de Venise et de Belmont, présentés en alternance avec leurs problématiques respectives, nous sont déjà, à ce stade, parfaitement familiers, si bien que les pitreries d'un clown risquent de retarder des échéances qui nous intéressent bien davantage. Le propos de Gobbo qui se demande s'il doit, s'il peut sans danger pour son âme quitter son maître, le Juif Shylock, pour se faire engager ensuite par Bassanio pourra paraître mal à propos. A moins bien entendu que ce petit intermède dramatique, véritable pièce dans la pièce, ne cristallise à travers les voix qu'il nous fait entendre 1'harmonie si particulière du Marchand de Venise.

Je voudrais montrer que cette modeste mise en scène de Gobbo entretient avec la comédie romanesque qui l'englobe un rapport fondamental. Non seulement le passage s'inscrit dans le mouvement général de Venise vers Belmont, non seulement il annonce la double désertion de Jessica, désertion filiale et religieuse, mais il s'inscrit grâce à la multiplicité des voix qui le composent dans la texture poétique profonde de la pièce. Cette petite scène, où il est 
crucial pour Gobbo de faire le bon choix, s'apparente, préfigure et parodie à l'avance les scènes des coffrets. Car même si Gobbo la présente comme une simple alternative entre rester ou partir, obéir à sa conscience ou obéir au démon, elle se joue quand même entre trois voix effectives, qui s'expriment en direct et se répondent. Si les voix des deux allégories sont uniformes et quelque peu figées, celle de Gobbo par contre, qui transparaît derrière ses marionnettes avant de s'affirmer dans la magistrale synthèse finale, est multiple.

La voix la plus immédiatement reconnaissable est bien sûr la voix de la scène médiévale, la voix de la Moralité et on peut se demander si Will Kempe jouait cet échange à trois voix en ventriloque ou comme un one-man show, s'il donnait la préférence à l'immobilité ou au mouvement, ou s'il combinait les deux aspects.

Par comparaison avec les enjeux financiers et sentimentaux de Venise et de Belmont, «the merry bond» d'une part et l'épreuve du choix des coffrets à laquelle doivent se soumettre Portia et ses prétendants d'autre part, il est vrai que la problématique de Gobbo paraît assez simpliste. Sa décision de quitter son maître n'engage que lui, elle n'a aucune incidence ni sur l'action, ni pour les autres personnages. Elle ne rencontre aucun obstacle puisque, ainsi qu'on l'apprend très vite, Shylock est tout disposé à se débarrasser d'un serviteur trop gourmand à son gré, ce qui lui permettra par la même occasion de jouer un bon tour au chrétien qui s'apprête à l'engager. C'est alors que, dans son dénuement d'homme seul face à un destin sans éclat, Gobbo se crée, ex nihilo, une scène capable de rivaliser avec les tempêtes, les hasards, les caprices de la Fortune ou les voies de la Providence qui régentent l'existence des autres Vénitiens. Il propulse son conflit sur la scène médiévale et le confie aux allégories traditionnelles, à savoir sa propre conscience qui l'incite à rester sur place et le Démon qui lui enjoint de fuir. Tiraillé entre ces deux voix qui se disputent son âme, Gobbo peut prendre le devant de la scène. C'est lui qui arbitre le temps de parole de chaque intervenant, il précise bien qui parle : «My conscience says» ou «says the fiend», plutôt trois fois qu'une, introduisant des variations dans l'ordre des mots «says my conscience». Puis il s'implique : "'Conscience' say I», «Fiend' say I». Le démon, quant à lui, nous prouve son authenticité en invoquant le ciel en vain: «For the heavens rouse up a brave mind» (11). Et pour ce qui est du programme, il est simple et précisé dès la première phrase: «Certainly, my conscience will serve me [dans le sens de «allow me»] to run from this Jew my master». Cependant l'allégorie de la 
conscience de Gobbo, tout heureuse d'exister, ne se laisse pas facilement subjuguer, elle tient bon, ce qui oblige le clown à utiliser des moyens rhétoriques pour le moins inattendus dans la bouche d'un homme aussi simple. De tels procédés mettraient d'ailleurs en doute son «honnêteté» foncière, dûment attestée, si cette inspiration quasi miraculeuse ne recouvrait une innocence qui elle aussi sonne juste. Deux voix distinctes se font entendre simultanément, il sera intéressant de rechercher leur origine.

Nous examinerons tout d'abord la voix du sophiste.

D'un côté il y a la voix insidieuse du Malin, qui a l'avantage d'offrir un programme concret en trois temps «use your legs, take the start, run away» (5). Ce programme est beaucoup plus original et attrayant que celui de cette conscience bornée qui s'obstine à répéter la même chose «take heed honest Launcelot, take heed honest Gobbo» (6-7) ou bien «do not run, scorn running» (8) ou encore un peu plus loin «budge not» (18-9). C'est une voix répétitive, dépourvue d'imagination et qui, nous le verrons, se laisse trop facilement envahir par une autre voix, celle de Gobbo luimême, lors entre autres exemples de la digression sur l'honnêteté parentale. Il est vrai que la voix du Malin s'épuise aussi assez rapidement et si ce dernier propose une action que l'on peut qualifier de positive «budge» pour la simple raison qu'elle n'est pas à la forme négative, on arrive quand même très vite à une impasse qui conduit Gobbo à prendre la parole pour faire la synthèse et tirer ses conclusions. C'est à ce stade que le sophiste entre en jeu. Il s'agit véritablement d'une entreprise de déstabilisation de l'auditeur témoin, parfaitement programmée en trois temps. Toute attente logique va se trouver radicalement bousculée. Tout d'abord, puisque Gobbo commence par dire à sa conscience "'Conscience' say I, 'you counsel well'» $(20)$, on attend bien entendu le pendant opposé lorsqu'il s'adresse au Malin. Pourtant que lui dit-il ? «'Fiend' say I, 'you counsel well'» (21). Il y a là matière à être désorienté. Mais sans doute Gobbo est-il déjà en train de ménager la chèvre et le chou en recouvrant sous la même formule des appréciations d'un ordre différent. Ce qu'il oppose en fait, c'est l'objectivité de la voix morale telle qu'il la conçoit à sa propre préférence subjective. La seconde étape nous entraîne au cœur même du sophisme lignes 21 à 25:

To be ruled by my conscience, I should stay with the Jew my master, who (God bless 
the mark) is a kind of devil ; and to run away from the Jew I should be ruled by the fiend, who (saving your reverence) is the devil himself. (21-5)

La structure en chiasme de l'argumentation endort la vigilance de l'auditeur attentif qui se trouve ainsi contraint d'adhérer à la conclusion, aussi pervertie soit-elle. La disposition des polarités est parfaite : la Conscience est opposée au Malin, l'alternative pour Gobbo est simple : ou bien rester chez le Juif ou bien fuir de chez le Juif, les inclusions entre parenthèses sont introduites par le même relatif «who». Ces inclusions, sortes d'exorcismes préventifs rajoutés par le porte parole Gobbo, conscient du rôle dangereux qu'il est appelé à jouer, font partie intégrante de la stratégie. Elles servent en fait d'apparents points de repère qui contribuent à nous égarer dans ce labyrinthe de pseudo-correspondances. Cherchons maintenant les deux différences. La première tourne autour de ce passif contraignant «to be rul'd», dont les voyelles longues et la dentale finale clouent sur place, clôturent l'horizon, ce projet si peu engageant par rapport à son homologue dynamique «to run away» dont la fluidité vocalique s'ouvre sur l'infini. La seconde différence porte sur le sens du modal. «I should stay» courbe l'échine et se tait sous le poids de l'obligation morale tandis que «I should be ruled» se perçoit comme une contrainte extérieure au sujet, comme une conséquence implicite qui s'impose mais le laisse libre. La voix naïve de Gobbo perce sous ces deux écarts, l'éblouissante sophistication du contexte lui étant en quelque sorte accordée juste pour cette circonstance exceptionnelle et providentiellement.

J'en arrive au troisième stade de déstabilisation rhétorique. Il porte sur les rapports entre «the Jew» et «the devil». Une première analogie s'établit pour présenter the Jew comme «a kind of devil» (23). Elle transite par l'assimilation entre «the fiend» et «the devil himself» (25) qui représente la deuxième étape contradictoire de l'argumentation. Celle-ci prépare la troisième étape qui culmine dans «the Jew is the very devil incarnation» (26), tant il est vrai que l'âme simple est toujours plus facilement impressionnée par la représentation concrète que par l'abstraction. Ainsi, pour étayer sa cause, Gobbo n'hésite pas à ajouter un chapitre à l'histoire religieuse. Il suggère que le diable aurait une nouvelle fois singé la trinité en envoyant sur terre son propre double à forme humaine, «the very devil incarnation», pour damner l'humanité. Shylock se profile soudain comme un antechrist avant la lettre. Gobbo pressent 
d'ailleurs que ses paroles ont un caractère subversif et c'est pour disperser l'odeur de soufre qu'il se signe entre parenthèses «God bless the mark» et en quatre syllabes. Mais c'est aussi ce qui fait pencher la balance du bon côté car Gobbo comprend très bien qu'entre deux maux et deux maudits, il est légitime de choisir le moindre : la voie est libre !

Il est certain que Gobbo place son dilemme sur un plan essentiellement moral et religieux et en cela il retrouve sans le savoir l'esprit de la source principale du Marchand de Venise. Il retrouve non seulement l'esprit mais aussi la voix de la «Gesta Romanorum».

Il semble que, dans sa quête, Gobbo paradoxalement courtcircuite la voie tracée par Shakespeare pour évaluer les prétendants. Il lui préfère la voie étroite traditionnelle suggérée par la Gesta Romanorum. En effet, on se souvient que les deux premières devises inscrites sur les coffrets d'or et d'argent sont reprises textuellement par Shakespeare dans un ordre inversé. La première est, rappelonsle, «Who so chooseth mee shall finde that he deserveth», la deuxième "Who so chooseth mee shall finde that his nature desireth». Cependant, la teneur de la troisième devise, celle du coffret de plomb qui contient le portrait de Portia, est de nature tout à fait différente. Alors que la source insiste sur la nature spirituelle mais codifiée du choix «Who so chooseth mee, shall finde that God hath disposed for him», la morale de l'histoire étant la récompense de la vertu, Shakespeare donne une autre portée à son histoire romanesque. Il conserve la notion de pèlerinage mais il la transpose dans le contexte amoureux. De même, il surqualifie la vertu qui devient plurielle et merveilleuse : Bassanio évoque les «wondrous virtues» (I.1.163) de Portia. La perspective dans la pièce de Shakespeare s'exprime davantage par rapport à un risque de nature existentielle : "Who chooseth me, must give and hazard all he hath» ${ }^{2}$. La rigide loi divine de la source est remplacée ici par la Providence. Or, au moment décisif où il s'apprête à faire son choix, Gobbo paradoxalement apparaît bien comme le seul des prétendants à réintroduire la perspective eschatologique, dans une forme il est vrai assez inattendue mais qui reste néanmoins très efficace. Bien sûr, Gobbo ne prétend pas à la main de Portia, il s'agit pour lui simplement de pouvoir prétendre suivre le Prétendant. Alors, semblable aux artisans du Songe d'une nuit d'été qui ne peuvent que singer leur créateur dans leur mise en scène de Pyrame et Thisbé, Gobbo se confectionne lui-même l'épreuve, le test décisif qui lui 
permettra d'accéder à Belmont comme serviteur de Bassanio. La mise en scène de la Moralité, fabriquée de toutes pièces par un clown contraint de prendre en main son destin puisque son créateur semble occupé ailleurs, n'est qu'un retour en arrière, une forme désuète, mais c'est en même temps une mise en abyme parodique et régressive de la vraie scène de choix dans Le Marchand de Venise.

Gobbo, grâce aux scrupules qui l'honorent même si personne n'en a que faire, s'introduit subrepticement, comme par hasard et presque à notre insu, dans la structure fondamentale du Marchand de Venise. Comme s'il était conscient que l'accès à Belmont doit se gagner, Gobbo se lance tout entier dans une entreprise d'une ampleur telle qu'elle ne saurait réussir sans l'aide inopinée de la Providence. Si bien que la voix que nous entendons lorsque Gobbo semble s'enferrer dans ses problèmes de conscience n'est autre que la voix du «Marchand de Venise».

Tout comme les artisans du Songe, le pauvre Gobbo pèche par excès, il en fait trop. Pourquoi s'inventer inutilement des scrupules, pourquoi s'inventer une conscience s'il faut ensuite la convaincre de se taire? S'inventer une conscience, n'est-ce pas s'inventer une âme que l'on risque aussi de perdre ? Et c'est justement là que Gobbo, en toute innocence, rejoint l'esprit de la troisième devise de Belmont. L'humble clown qui vient de se doter d'une âme doit assumer ce risque et la remettre immédiatement en jeu, ce qui revient à «donner et hasarder tout ce qu'il a» ${ }^{3}$. Le clown manipulateur se trouve ainsi comme repris en sous-main par son auteur. Il rejoint pour de bon, sans le savoir, la troupe des aspirants au départ et se qualifie pour le voyage à Belmont. Gobbo, confiant, espère d'ailleurs déjà à la fin de la scène que sa fortune sera bonne et qu'elle aura un visage de femme ${ }^{4}$.

Le serviteur de Shylock, en tant que tel, n'était nullement qualifié pour le monde idéal de Belmont. En passant au service de Bassanio, Gobbo fait bien davantage que «quitter le service d'un riche Juif pour se mettre à la suite d'un pauvre gentilhomme» (1401). Il change non seulement de livrée mais de voix. Pour gagner les galons de cette nouvelle livrée, Gobbo devra aussi se qualifier comme clown d'esprit. $\mathrm{Si}$, dans l'atmosphère asphyxiante de la maison de Shylock, sa voix résonnait plus ou moins dans le vide ${ }^{5}$, c'est en principe sa capacité verbale qui va faire de lui le clown d'un gentilhomme. C'est à la voix qu'il sera jugé. Mais, nouveau paradoxe, c'est pourtant Gobbo qui assure lui-même son salut, non 
par la nouvelle fonction d'amuseur professionnel à laquelle il sera finalement promu, mais en solitaire, par le biais d'une volubilité aussi savante que pervertie. La décision favorable de Bassanio ne fera que confirmer ce qui est déjà gagné à la fin du monologue. Shakespeare, ou la Providence, extirpe Gobbo de l'impasse dans laquelle il s'est malencontreusement enferré en le gratifiant d'une rhétorique dont la perfection formelle n'a d'égale que le caractère absolument spécieux de l'argumentation. Ce monologue est un chef d'œuvre d'aveuglement qui se joue en duo : il est à la fois soufflé, insufflé à Gobbo et naïvement assumé par lui. Ici peut se poser la question de la nature exacte du sophisme déjà évoqué. S'agit-il d'un authentique sophisme ou d'un paralogisme? Y a-t-il volonté délibérée de tromper le destinataire du message ou non ? La voix étant double, la réponse l'est aussi. Nous avons là un paralogisme doublé d'un sophisme. La voix porte-parole qui se contente de dire tout haut ce que la voix salvatrice providentielle lui suggère utilise l'argument comme un paralogisme. Elle en est la première victime et bénéficiaire reconnaissante. Par contre, il est certain que le casuiste qui l'inspire pourrait difficilement sortir Gobbo de l'impasse sans avoir recours au détournement délibéré du sophisme. Pour Gobbo en effet, c'est une question de survie car ce n'est qu'en désobéissant à sa conscience qu'il pourra accéder à son nouveau statut de clown libre. En l'occurrence, il rejette le plomb, la chape à défaut de coffret. Ce faisant, il répond du même coup aux exigences de sa fonction puisque le clown, par définition fait tout à l'envers. Si la sagesse peut se lire dans la folie, pourquoi le salut ne se lirait-il pas dans la transgression ? ${ }^{6}$

Gobbo réussit à se sauver dans les deux sens du terme. Il sauve son âme de clown parce qu'il se sauve de chez son maître Shylock. S'il y parvient, c'est en accord avec ses exigences intimes, des exigences qui l'ont conduit à résoudre sur la scène publique, en toute apparente bonne foi, les contradictions qui l'assaillaient. Nous allons essayer d'écouter à présent les voix intimes de Gobbo qui s'expriment dans les méandres du texte.

Au départ, il faut prêter l'oreille pour entendre, à travers ce qui est dit, la voix de Gobbo sous la chape de plomb. Nous avons vu que les deux allégories remplissent très honnêtement leur rôle, le démon tentateur en appelle au courage, «rouse up a brave mind» (11) et la conscience à la sagesse, «very wisely» (13). Refusant d'être juge et partie, Gobbo s'applique à écouter l'une puis l'autre. Cependant, c'est quand même lui, Gobbo, qui présente les deux forces 
contradictoires et il les représente de façon picturale. Lè démon est là sur le côté, qui le tire par le coude, insistant mais distinct. La conscience, par contre, envahit sans ambages son intimité, pénètre comme en terrain conquis jusqu'à son cœur, va jusqu'à se pendre au cou de son cœur : «hanging about the neck of my heart» (13). L'expression employée ici par Gobbo est une image d'origine biblique qui figure en bonne place dans les Évangiles ${ }^{7}$, dans le discours du Christ sur le scandale tel que le rapportent saint Matthieu, saint Marc et saint Luc. Il est facile de la compléter, c'est en fait «hanging like a millstone around someone's neck», avec cette image de la meule que l'on met au cou du coupable avant de le précipiter dans la mer. Cette expression fait en effet référence au supplice de la submersion avec une meule au cou. Elle est passée dans le langage courant avec un sens figuré et elle exprime alors l'idée de paralysie, d'accablement en cas d'angoisse extrême. L'image originelle évoque le poids de la pierre taillée dans la masse, matière tout aussi élémentaire et tout aussi lourde que le plomb. D'ailleurs, si on cherche à la traduire en français, cela donne «comme une chape de plomb» en référence à une autre forme de supplice, en usage au Moyen Âge, celui du manteau de plomb dont on revêtait les condamnés qui mouraient ainsi par lente suffocation s'ils n'étaient pas auparavant jetés dans les flammes ${ }^{8}$. On retrouve, par analogie, le vil métal et la voix de Gobbo étouffée, suppliciée sous son poids, dans le filigrane de la description. D'un écho poétique à un autre, on se souvient que dans l'Enfer de Dante, ce sont les «tristes hypocrites» qui succombent sous le poids des chapes dont l'endroit brille comme de l'or tandis que l'envers n'est que vil plomb ${ }^{9}$.

Gobbo, cependant, a encore les pieds sur terre et il est évident qu'une certaine ambiguïté se niche au cœur même de la voix liée si étroitement à l'image de la meule. Il exprime, de toute évidence, son sentiment de culpabilité à travers cette image entendue depuis la chaire à l'église, mais en même temps, pour neutraliser ses effets, il la retourne, il la détourne en la sécularisant. Ainsi, le coupable se métamorphose-t-il en victime. C'est pourquoi il oublie de montrer la meule et exhibe à sa place son propre coeur, «the neck of my heart», le symbole de sa propre chair et du siège de la souffrance. C'est la livre de chair offerte en sacrifice, mise de nouveau en jeu. C'est aussi la bifurcation vers l'humain qui, en l'occurrence, mène au salut. Et Gobbo après tout n'a rien à se reprocher car même si la tentation est trop forte, il peut quand même en toute impunité effectuer ce qu'on pourrait appeler «un 
retour en arrière sur projection conditionnelle par le biais d'un détournement de sens» ${ }^{10}$. Il peut préférer en fin de compte le sens figuré au sens littéral, puisque sa culpabilité n'est pas encore confirmée et qu'il reste maître du jeu.

De toute évidence, Gobbo est plus à l'aise lorsqu'il évoque les faits de la vie courante. La voix de l'honnête homme qui ne s'en laisse pas compter et qui se montre soucieux de faire entendre la vérité s'affirme bientôt. Gobbo écoute ses voix mais certainement pas d'une façon passive. Lorsqu'en accord parfait avec une conscience qui le flatte, il entend la voix populaire adepte du lieu commun et qui aime faire de l'honnête homme le fils d'un honnête homme, Gobbo par honnêteté pointilleuse intervient. Dans son cas particulier, il s'agirait d'une contre-vérité qui se doit d'être signalée. On prendra donc le numéro deux sur la liste des bénéficiaires, ce qui donne, à défaut d'un «honest man's son», «an honest woman's son» (15).

De fil en aiguille, les écarts de conduite de Gobbo père pourraient aisément donner lieu à un développement improvisé, toujours fertile et attendu pour la traditionnelle voix scandaleuse et facilement licencieuse ${ }^{11}$ du clown, mais ce n'est ni le lieu ni l'heure. Gobbo s'autocensure soudain et ses deux petites phrases inachevées en suggèrent long, puis il reprend le ton sérieux et docte qui convient «well, my conscience says...» (17).

Si la virtuosité de ses sophismes peut laisser supposer qu'une force supérieure bienveillante s'amuse à tirer parfois les ficelles du pauvre Gobbo, la conclusion du monologue, par contre, nous replonge dans une tonalité mieux accordée au simple mortel qu'il demeure. Les contradictions qui le tourmentent sont toujours aisément identifiables mais elles se sont chargées de connotations subjectives. On retrouve d'un côté «a kind of hard conscience» (27) s'opposant au «more friendly counsel» (29). A présent Gobbo perçoit une dissonance certaine entre sa conscience intime, la première de ces deux consciences qu'il revendique comme siennes, et cette seconde conscience, intransigeante et lointaine, qu'il s'est imposée : «in my conscience, my conscience is but a kind of hard conscience». Gobbo se sent de toute évidence plus proche de la première, bien plus en phase avec cette petite voix familière. La seconde devient une sorte d'avatar mal défini et cruel «a kind of hard conscience» (27). Il y a rupture tonale et totale, désaccord parfait lui-même prélude à un changement de tonalité, une modulation en bonne et due forme. La conscience se voit par la même occasion privée définitivement du droit de réponse tandis 
qu'un Gobbo impatient et résolu s'accorde le temps de clamer par deux fois «I will run»! Emblématiques du transfert d'allégeance, les talons soumis au diktat de la conscience ligne $9^{12}$ se mettent instantanément au diapason et au service du démon : «My heels are at your commandment» $(30)$.

La petite conscience de Gobbo remplit l'espace vocal mais sa tonalité jubilatoire trahit une inflexion secrète qui ne sera dévoilée qu'un peu plus tard, dans la seconde tirade considérée ici (149-60). Ce passage éclaire et justifie l'infléchissement du discours initial vers sa totale libération. Cette partie pourrait s'intituler la voix des sirènes de Belmont.

Parvenu avec un peu d'aide à se libérer de l'enfer Shylockien, à accéder à un statut supérieur de clown de bonne compagnie, Gobbo peut enfin laisser libre cours à ses aspirations profondes et révéler cette ambition intime, inscrite en creux dans l'énergie phénoménale déployée lors de son ordalie. Dans le monde romanesque, il n'est pas rare que l'amour d'une femme, d'une Béatrice ou d'une Guenièvre, implique pour le héros la traversée littérale ou métaphorique de l'enfer. La femme idéale de Gobbo reste anonyme et si peu définie qu'elle en est universelle. Surabondante, elle allie l'expérience de la veuve à l'innocence de la vierge. Le lit de plume qu'elle fait miroiter est loin d'être sans risques. Si l'on peut parler d'intervocalité, on voit que Gobbo et Touchstone partagent le même registre. Leurs désirs se tournent tout naturellement vers l'amour dans le mariage ${ }^{13}$. Il est vrai que la «petite quinzaine de femmes» (153) de Gobbo, les «onze veuves et neuf vierges» (154) virtuelles ne vaudront jamais une Audrey en chair, en os et en prose. Mais le désir est bel et bien exprimé. Le fait qu'il reste à réaliser n'enlève rien à sa valeur symbolique et surtout à sa fonction essentielle de contrepoint par rapport à l'amour idéalisé de Belmont ${ }^{14}$.

En conclusion, que nous disent les voix de Gobbo ? Elles nous précisent la position du locuteur par rapport à son projet. L'apparent dilemme est en réalité un véritable coup de force. Il est sûr que la résolution sincère d'un authentique cas de conscience aurait orienté le discours vers l'intériorité discrète du soliloque. A l'inverse, ce monologue résonne de toutes ses voix vers l'extérieur. La multiplication de ces voix permet à celui qui les orchestre d'en privilégier une. On reproche parfois à Gobbo d'être le moins accompli des clowns de Shakespeare, je pense toutefois que sa voix 
- en particulier dans ce morceau de bravoure - mérite d'être entendue. Méritoire est la quête forcenée du risque que poursuit, pour aussitôt formulé s'y soustraire, ce Lancelot de la Lagune. Et c'est finalement la voix de Dogberry, son frère, son double, qui nous donne indirectement la clef du monologue et résume à merveille le parcours inouï de Gobbo dans ce texte. L'intrépide Lancelot ne vient-il pas, en effet, de découvrir la voie, tout autant que la voix, qui permet de se condamner soi-même à la «Rédemption éternelle» ${ }^{15}$ ?

Claudine DEFA YE Université de Poitiers

\section{NOT E S}

1 Lorsque le téléfilm de la $\mathrm{BBC}$ a été programmé sur une chaîne française en 1985, les scènes de Gobbo ont été systématiquement supprimées.

${ }^{2}$ The Merchant of Venice, II.7.9.

${ }^{3}$ L'idée de risque est reprise par Gobbo lui-même dans les vers 98-9: «as I have set up my rest to run away, so I will not rest till I have run some ground» sous la forme du jeu de cartes appelé Primero. La note de l'édition Arden précise que cette expression signifie «to venture one's final stake or reserve».

${ }^{4}$ Cf. II.1.152 \& 158 : «I shall have good fortune» et «if Fortune be a woman».

${ }^{5}$ Jessica fait allusion au faible écho de la voix de Gobbo dans la scène suivante :

Our house is hell, and thou (a merry devil)

Didst rob it of some taste of tediousness, - (II.3.2-3)

${ }^{6}$ Gobbo qualifie le démon de «most courageous fiend» (9) comme s'il redonnait à celui-ci, à présent englué dans son rôle routinier de tentateur, la qualité première qui l'a conduit à se séparer de Dieu et à se damner. Gobbo trahit peut-être un sentiment d'identification avec le symbole de la Tentation originelle au moment même où il s'apprête lui aussi à franchir le pas décisif.

${ }^{7}$ Cf. Évangile selon saint Matthieu (18.6): «Mais si quelqu'un doit 
scandaliser l'un de ces petits qui croient en moi, il serait préférable pour lui de se voir suspendre autour du cou une de ces meules que tournent les ânes et d'être englouti en pleine men». Cf. aussi Évangile selon saint Marc, IX.42 et Évangile selon saint Luc, XVII.

${ }^{8}$ Selon De Ste Foix (Ess. Hist. 3e part. p 72), Jean Sans Terre fit ainsi périr l'archidiacre de Norwich.

${ }^{9}$ Dante Alighieri, La Divine comédie, Chant XXIII.

${ }^{10}$ Dans une étude parallèle sur «Le détour», qui s'intitule «Détours et bouffonneries dans trois comédies romanesques de Shakespeare», à paraître dans La Licorne, Publications de l'Université de Poitiers, Hors-Série, printemps 2000, j'explicite cette notion en ces termes : «Gobbo sent faiblir sa résistance morale et anticipe le châtiment qui va inéluctablement en résulter. C'est pourquoi l'image première qui surgit spontanément dans son esprit exprime son sentiment de culpabilité. Cependant, il effectue, tout aussi spontanément, un transfert vers l'image devenue populaire et séculière de la victime assaillie par une force extérieure encombrante. La forme tronquée de l'image, dans laquelle la meule est évacuée, replace le dilemme sur un plan pratique et familier plus facile à résoudre pour l'homme simple qu'est Gobbo. Gobbo se simplifie la vie en quelque sorte. Le sens originel est étouffé dans l'œuf au profit du sens figuré qui relativise la faute avant même qu'elle ne soit commise».

${ }^{11}$ La note de l'édition Arden signale que «grow to» a probablement une connotation paillarde.

12 Cf. vers $8-9$ : «scorn running with thy heels».

${ }^{13}$ Touchstone se contente d'une seule épouse mais il se réserve une porte de sortie : cf. As You Like It : «I were better to be married of him than of another, for he is not like to marry me well ; and not being well married, it will be a good excuse for me hereafter to leave my wife» (III.3.81-5). Gobbo rêve plutôt d'un harem mais il entrevoit également les risques encourus : «and to be in peril of my life with the edge of a feather-bed» (156-7).

${ }^{14}$ C'est encore Touchstone qui exprime le mieux cette philosophie realiste: «As the ox hath his bow, the horse his curb, and the falcon her bells, so man hath his desires» ( $A Y L$, III.3.71-2).

${ }^{15}$ Cf. Much Ado About Nothing, IV.2.53-4. 